ISSN 1112-9867

http://www.jfas.info

\title{
LINEAR AND NON-LINEAR SIMULATION OF JOINTS CONTACT SURFACE USING BEAM-LIKE COMPONENT
}

\author{
A. Chonnani Mohisanzadeh ${ }^{1}$, H. Jalali ${ }^{2}$ and M. Rajaei ${ }^{3}$ \\ ${ }^{1} \mathrm{MS}$ student of Arak Industrial University \\ ${ }^{2}$ Associated professor at Arak Industrial University \\ ${ }^{3}$ Assistant professor at Arak Industrial University
}

Published online: 15 May 2016

\begin{abstract}
The joint modelling including non-linear effects needs accurate and precise study of their behaviors. When joints are under the dynamic loading, micro, macro- slip happens in contact surface which is non-linear reason of the joint contact surface. The non-linear effects of joint contact surface on total behavior of structure are measured by various criterions such as frequency response diagram and hysteresis loop. The objective of this research is first studying a two freedom degree system including non-linear component of hysterical valance to show its non-linear effects on structure behavior. Later, a non-linear contact model called beam-like component was simulated by ANSYS software was used amplitude to simulate joints contact surface of cantilever beam. Being linear or non-linear of this model was proved by various criterions such as frequency response diagram, hysteresis loop, and energy dissipation diagram with excitation force. The results show acceptable accuracy and modelling precision.
\end{abstract}

Keywords: micro-slip, non-linear joints, finite elements, beam-like component

Author Correspondence, e-mail: Seji1991@yahoo.com

doi: http://dx.doi.org/10.4314/jfas.v8i3s.239 


\section{INTRODUCTION}

Most fabricated engineering structures have been made by different joints pieces such as bolts and cap screws, riveter, welding, glue, cantilever, etc. the structure stiffness and also natural frequencies, damping, and non-linear behaviors are significantly dependent on nature and joints points. Particularly, in metal structures, the occurred damping by joints is relatively more than materials damping [1]. The factors such as preload force, external force, energy dissipation, micro-slip range, and stick-slip must be certainly mentioned. Each mentioned factors influence joints behavior in a particular way. Joints has vital role to examine accurate dynamic behavior of a structure including joints, because these types of joints are with frequencies damping and energy dissipation. These pieces may be turbine blades, space missions, or military installations. These structures are influenced by dynamic loads and high frequencies and greatly influence on joints, and may make joints behaviors non-linear [2]. These non-linear behaviors are in micro-slip in one joint. It means they slip in some pint of interface. Various models including dry friction models, semi-hysteric models, and hysteric models are used for dynamic modelling of these micro-slips. The objective of this research is providing a model to be able of accurate modelling of these linear and non-linear effects and micro-slips and stick-slips. Since some joint containing and sophisticated pieces have no condition of experiment or high cost experiments, a simulated software model for a real joint is necessary whose behavior is near to a real joint. Many studies were conducted by Ferri about joints modelling in 1995 by resulted damping from friction [3].Berger (2002) modelled friction for system dynamic solution. [4] Ibrahim and Pettite (2005) worked on bolts and other joints dynamically [5]. Various models have been suggested to detect the joint interface mechanisms in past years. Amongst, the micro-slip models are well-known that let partial slip in some points of interface. Iwan model [6] is usually used for micro-slip modelling and is made from parallel springs in series with Colomb friction components. This model is known as an excellent plastic-elastic model. There are some other models suggesting stick-slip to micro-slip and macro-slip stares. The Valance model [7, 8, 9, 10], LuGre model [11], Dahl model [12], Bouce-Wen model [13], and Leuven model [14, 15] are samples of this modelling. Mayer ad Gual (2005) worked on bolt joints non-linear modelling using finite-element 
software that simulates joint to a thin non-linear later. [16] Jalali et al. (2011) detected and modelled non-linear joints parameters using thin layer model [17].

\section{Non-Linear Freedom Degree Two Model}

Freedom degree two system was analyzed ad modelled in this part. Therefore, a freedom degree 2 system with joints was analyzed that this joint was modelled with a non-linear component. The freedom degree 2 schematic is shown in figure (1) which is used in numerical example. This system includes a loaded non-linear component by a harmonic excitation.

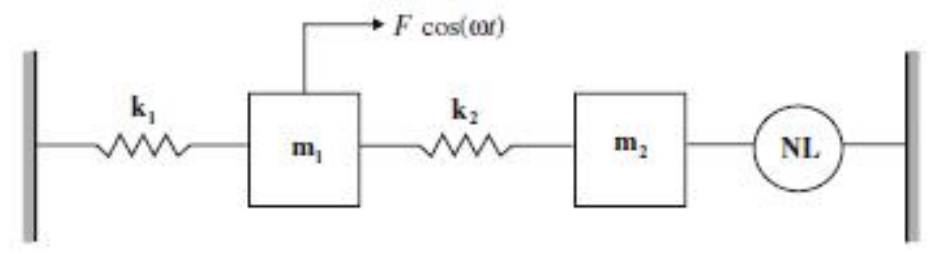

Fig.1. Non-linear freedom degree 2 system

Consider a non-linear freedom degree 2 system with forced excitation of figure (1) that is connected to non-linear component with mass $m_{2}$ with 2 linear springs of $k_{1}, k_{2}$ and masses $m_{1}, m_{2}$. The oscillation movement equation corresponding to this system is as following:

$\left[\begin{array}{ll}m_{1} & 0 \\ 0 & m_{2}\end{array}\right]\left\{\begin{array}{l}\ddot{x}_{1}(t) \\ \ddot{x}_{2}(t)\end{array}\right\}+\left[\begin{array}{cc}k_{1}+k_{2} & -k_{2} \\ -k_{2} & k_{2}\end{array}\right]\left\{\begin{array}{l}x_{1}(t) \\ x_{2}(t)\end{array}\right\}+\left\{\begin{array}{l}0 \\ f_{N L}\end{array}\right\}=\left\{\begin{array}{l}f \\ 0\end{array}\right\} \cos (\cot )$

$\ddot{x}_{1}(t), \ddot{x}_{2}(t)$ are first and second derivatives of system response to time, respectively. $f_{N L}$ is non-linear force imposed to mass $m_{2}$. In order to solve a non-linear system, first it should change into a linear system and then the mentioned response in extracted. The theoretical solution is not recommended for non-linear systems for its length and sophistication of non-linear oscillation systems. The numerical methods are used to solve the non-linear systems. If $[K]$ is stiffness matrix, $[M]$ is mass matrix, and $\left\{F_{L, N L}\right\}$ is linear and non-linear force vector of the above system, the equation (1) can be written as: 


$$
\left.[M]\{\ddot{x}(t)\}+[K]\{x(t)\}=\left\{F_{L, N L}\right\} \quad\right) 2(
$$

Equation (2) is freedom degree 2. In order to solve this equation, it should change into freedom degree 1 equation;

$$
[A]\{\dot{q}\}+[B]\{q\}=\{F\}
$$

In which, matrices $\mathrm{A}, \mathrm{B}$, and $\mathrm{F}$ are as following;

$$
\left.[A]=\left[\begin{array}{cc}
{[M]} & {[0]} \\
{[0]} & -[I]
\end{array}\right] ;[B]=\left[\begin{array}{cc}
{[0]} & {[K]} \\
{[I]} & {[0]}
\end{array}\right] ;\{F\}=\left\{\begin{array}{c}
\{0\} \\
\left\{F_{L, \mathrm{NL}}\right\}
\end{array}\right\} \quad\right) 4(
$$

$\{\dot{q}\},\{q\}$ are vectors are velocity and movement vectors of new coordinates that include velocity, movement, acceleration, and velocity of the previous coordinates, respectively; and is unit matrix:

$$
\{\dot{q}(t)\}=\left\{\begin{array}{l}
\{\ddot{x}(t)\} \\
\{\dot{x}(t)\}
\end{array}\right\} ;\{q(t)\}=\left\{\begin{array}{l}
\{\dot{x}(t)\} \\
\{x(t)\}
\end{array}\right\}
$$

) 5(

According to equation (3), it is obtained:

$$
\left.\dot{q}(t)=[A]^{-1}(\{F\}-[B]\{q(t)\}) \quad \quad\right) 6(
$$

The system response is obtained by solving equation (6) by Runge-Kutta method.

\section{Numerical Example}

Figure (1) shows s freedom degree 2 system with noon-linear component. The non-linear component follows equation (7):

$$
\left.f_{N L}=k_{3} x_{2}(t)+c_{1} \dot{x}_{2}(t)+\dot{F}_{V} \quad\right) 7(
$$

The Valance model has been used for non-linear component modelling whose restoring force, $\dot{F}_{V}$ is as equation (8):

$$
\dot{F}_{V}=\frac{e_{\mathrm{o}} V\left[1+\frac{\lambda}{e_{\mathrm{o}}} \operatorname{sgn}(v)\left(e_{t} x-F_{V}\right)\right]}{1+k \frac{\lambda}{e_{\mathrm{o}}} \operatorname{sgn}(v)\left(e_{t} x-F_{V}\right)}
$$

That $x$ is relative displacement and $v$ is relative velocity ad the first derivatives than time. 
$\operatorname{sgn}(v)$ Function is sign function and is defined as $\frac{v}{|v|}$, when $v \neq 0$. The dimensionless parameter $\lambda$ is define as equation (9):

$\lambda=\frac{e_{\circ}}{\alpha_{\circ}\left(1-k \frac{e_{t}}{e_{\circ}}\right)}$

) 9(

$e_{\circ}, e_{t}$, and $\alpha_{\circ}$ are Valance model stiffness parameters that control hysteresis shape and size.

The dimensionless parameter $\mathrm{K}$ shows adhesiveness transfer to slip way. The value 0.99 shows quick transfer from adhesiveness to slop state and less than 0.99 shows soft transfer.

System response according to figure (2) is shown for excitation amplitude and frequency of $\omega=8.5 \mathrm{rad} / \mathrm{s}, \mathrm{F}=60 \mathrm{~N}$ by solving equation (1).

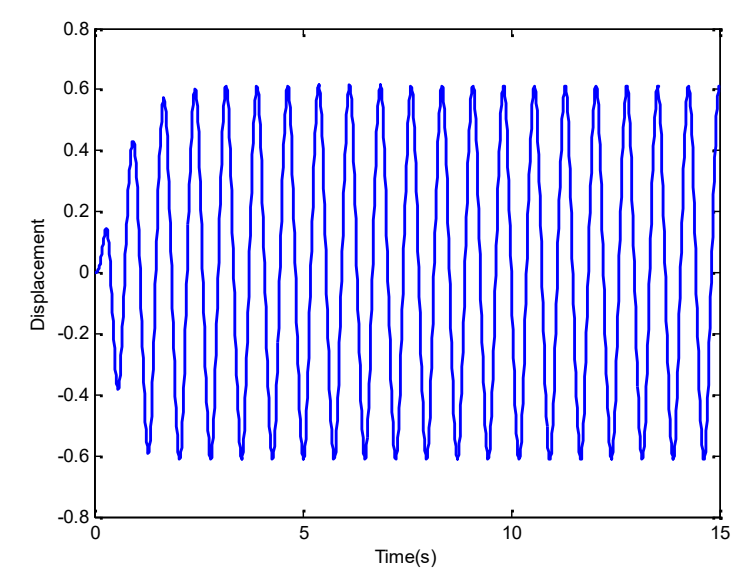

Fig.2. System response for excitation amplitude and frequency of $\omega=8.5 \mathrm{rad} / \mathrm{s}, \mathrm{F}=60 \mathrm{~N}$

In order to calculate system frequency response, first system response is calculated according to previous part for a certain frequency range, then the maximum amplitudes of these replacements than frequency range are drawn.

$$
\left.X_{i}(t) \approx X_{i}\left(\omega t+\psi_{i}\right), i=1,2 \quad\right) 10(
$$

$\mathrm{X}$ is response amplitude and is phase difference between response signals and excitation force. The frequency responses are determined using equation (11) by having system 
harmonic responses:

$\left.\alpha_{i 1}(\omega)=\frac{X_{i}}{F} e^{j \psi_{i}} \quad i=1,2 \quad\right) 11($

Figure (3) shows linear and non-linear frequency responses diagram for different excitation force amplitudes around the first frequency. As it is clear from figure, it is shown how non-linear component changed system behavior. The softening phenomenon happens in this state and curve converted to left side for non-linear Valance component, and frequency response tended to down side for damping in frequency response diagram system.

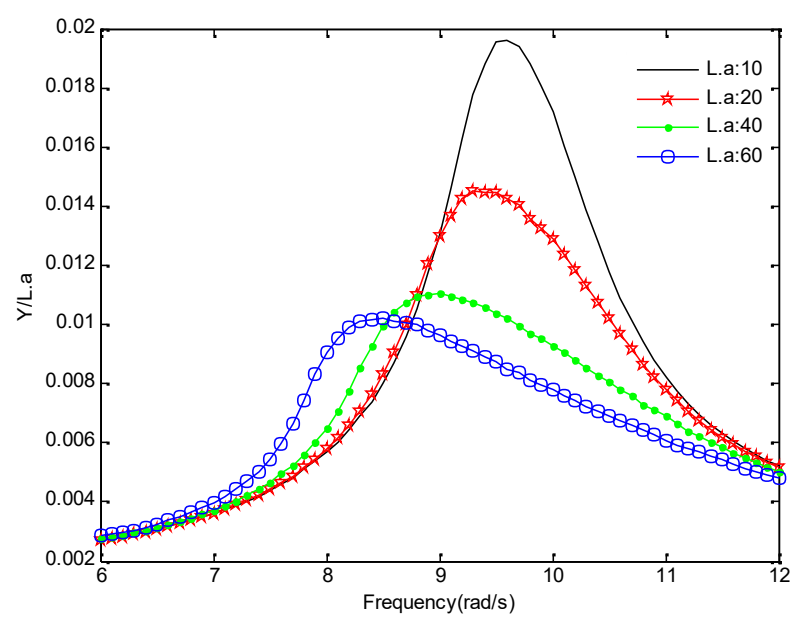

Fig.3. Linear frequency response diagrams with (-)10 excitation force amplitude, $\left.{ }^{*}\right) 20$ non-linear with excitation force amplitude, (•) 40 excitation force amplitude, and (o) 60 excitation force amplitude

The hysteresis loop of Valance model is shown in figure (4). The internal area of hysteresis loop shows energy dissipation and also adhesiveness-slop area of contact surface. 


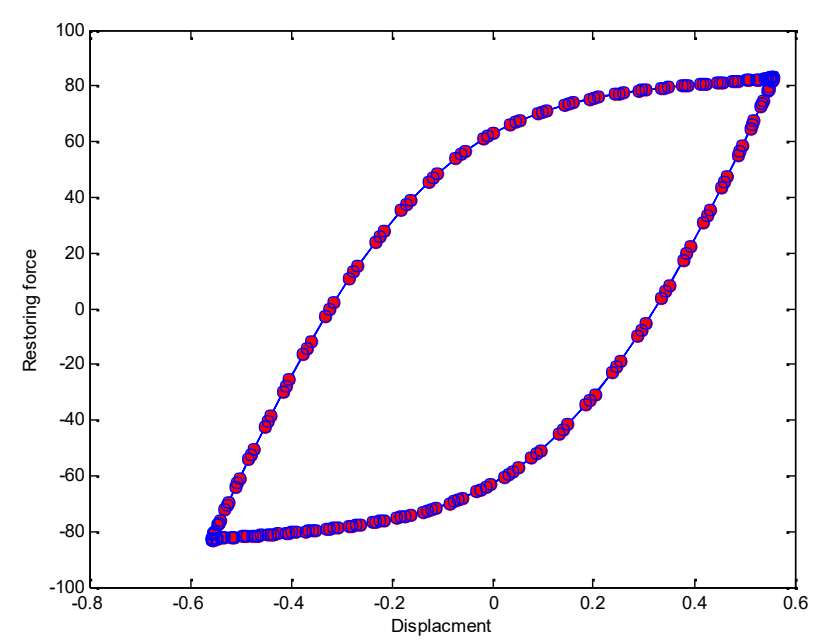

Fig.4. The hysteresis loop of Valance model with 40 excitation force amplitude and $9 \mathrm{rad} / \mathrm{s}$ excitation frequency

The Beam-Like Component model for Contact Surface

In this research, dynamic behavior modelling of joints contact surface is studied in a structure. This joints may be bolt or other connections. The used method in this chapter is simulation joint contact surface behavior. Thee contact surface behavior is simulated using beam-like component according to figure (5); the modelling was for both linear and non-linear states.

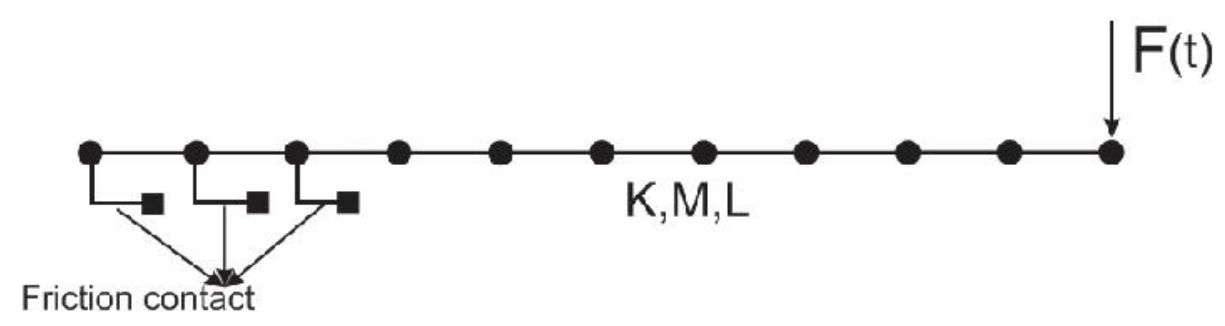

Fig.5. cantilever beam

Linear and Non-Linear Theory for Beam-Like Component

The joint contact surface of this modelling is by beam components (link) in $\mathrm{x}$ direction (tangential) and $\mathrm{K}$ components in y direction (normal) at the end of Euler-Bernoulli beam. Figure (6) show an element of contact surface which is made from beam element with cross section (A), length (L), Elasticity module (E), and element (K). The $F_{N L}$ element in linear form follows equation (12): 


$$
F_{L}=k_{s} y+c_{1} \dot{y}
$$

Erreur ! Il n'y a pas de texte répondant à ce style dans ce document.(

In which, $c_{1}$ is linear damping, and $k_{s}$ is element stiffness coefficient.

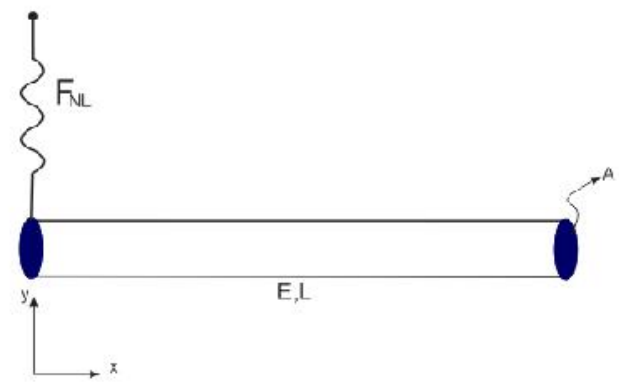

Fig.6. Contact surface element

If element $F_{N L}$ is fig (6) is designed in horizontal direction:

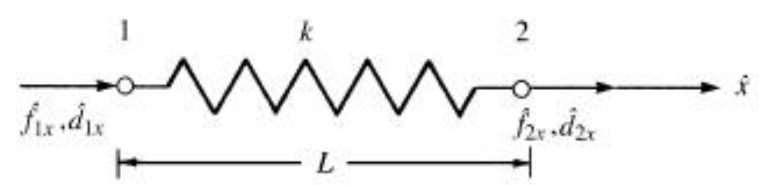

Fig.7. The linear spring element with node replacement and positive force distribution [18] The spring in figure (7) changes form after imposing force.

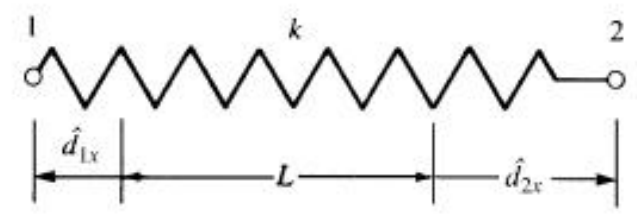

Fig.8. The changed spring formation

The relation of force of this element form change is as equation (12):

$$
T=k \delta \quad 12(
$$

That $\mathrm{T}$ is force and $\delta$ is spring replacement:

$$
T=k\left(\widehat{d_{2 x}}-\widehat{d_{1 x}}\right)
$$

By adding equilibrium: 


$$
\widehat{f}_{1 x}=-T, \widehat{f}_{2 x}=T
$$

By adding damping to figure (8) and passing the mentioned steps, the imposed force for both nodes will be as following:

$$
\begin{aligned}
& \hat{f}_{1 x}=k\left(\hat{d}_{1 x}-\hat{d}_{2 x}\right)+c\left(\hat{\dot{d}}_{1 x}-\hat{\dot{d}}_{2 x}\right) \\
& \hat{f}_{2 x}=k\left(\hat{d}_{2 x}-\hat{d}_{1 x}\right)+c\left(\hat{\dot{d}}_{2 x}-\hat{\dot{d}}_{1 x}\right)
\end{aligned}
$$

If equation (15) is stated in matrix form:

$$
\left.\left\{\begin{array}{l}
\hat{f}_{1 x} \\
\hat{f}_{2 x}
\end{array}\right\}=\left[\begin{array}{cc}
k & -k \\
-k & k
\end{array}\right]\left\{\begin{array}{l}
\hat{d}_{1 x} \\
\hat{d}_{2 x}
\end{array}\right\}+\left[\begin{array}{cc}
c & -c \\
-c & c
\end{array}\right]\left\{\begin{array}{l}
\hat{\dot{d}}_{1 x} \\
\hat{\dot{d}}_{2 x}
\end{array}\right\} \quad\right) 15(
$$

In figure (7) element, $x=y$.

Now, the beam element theory is examined. [18] The relation for replacement strain for element is as figure (9):

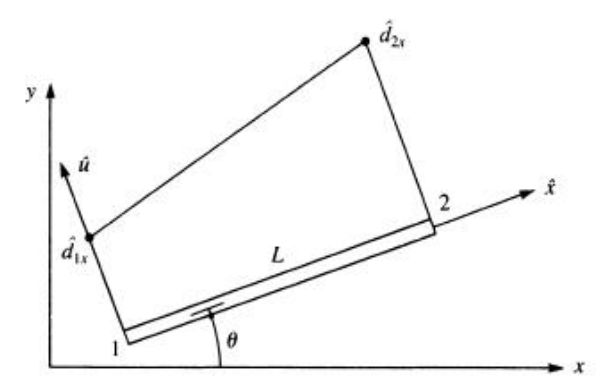

Fig.9. beam element [18]

$$
\varepsilon_{x}=\frac{d u}{d x}=\frac{d_{2 x}-d_{1 x}}{L}
$$

According to Hooke's Law for linear element:

$$
\sigma_{x}=E \varepsilon_{x}
$$

The imposed axial force to element is as following;

$$
T=A \sigma_{x}
$$

It is obtained from relations (16), (17), and (18): 


$$
T=A E\left(\frac{d_{2 x}-d_{1 x}}{L}\right)
$$

After inserting equilibrium:

$$
\left\{\begin{array}{l}
f_{1 x} \\
f_{2 x}
\end{array}\right\}=\frac{A E}{L}\left[\begin{array}{cc}
1 & -1 \\
-1 & 1
\end{array}\right]\left\{\begin{array}{l}
d_{1 x} \\
d_{2 x}
\end{array}\right\}
$$

According to relations (15) and (20), the linear element stiffness matrix is extracted as relation (21):

$$
\bar{K}=\left[\begin{array}{cccc}
\frac{A E}{L} & 0 & -\frac{A E}{L} & 0 \\
0 & k & 0 & -k \\
-\frac{A E}{L} & 0 & \frac{A E}{L} & 0 \\
0 & -k & 0 & k
\end{array}\right]
$$

If system becomes non-linear, the normal direction elements follow the following relation:

$$
F_{N L}=k_{s} y+c_{1} \dot{y}+c_{2} \dot{y}^{2}
$$

That $c_{2}$ is non-linear damping of element.

The beam element doesn't follow Hooke's Law in non-linear state and can be a non-linear function of stress than strain:

$$
\sigma_{x}=k \varepsilon_{x}^{n}
$$

The power $\mathrm{n}$ is different for each material and is obtained from experiment.

Now, if the previous steps are repeated for non-linear state, they will lead to non-linear stiffness of contact surface.

The force replacement relations for beam element are stated as following:

$$
\begin{aligned}
& f_{1 x}=k \frac{A E}{L}\left(d_{1 x}-d_{2 x}\right)^{n} \\
& f_{2 x}=k \frac{A E}{L}\left(d_{2 x}-d_{1 x}\right)^{n}
\end{aligned}
$$

And linear equation (15) changes to non-linear form: 
$\left\{\begin{array}{l}\hat{f}_{1 x} \\ \hat{f}_{2 x}\end{array}\right\}=\left[\begin{array}{cc}k & -k \\ -k & k\end{array}\right]\left\{\begin{array}{l}\hat{d}_{1 x} \\ \hat{d}_{2 x}\end{array}\right\}+\left[\begin{array}{cc}c_{1} & -c_{1} \\ -c_{1} & c_{1}\end{array}\right]\left\{\begin{array}{l}\hat{\dot{d}}_{1 x} \\ \hat{\dot{d}}_{2 x}\end{array}\right\}+\left[\begin{array}{cc}c_{2} & -c_{2} \\ -c_{2} & c_{2}\end{array}\right]\left\{\begin{array}{l}\hat{\dot{d}}_{1 x} \\ \hat{\dot{d}}_{2 x}\end{array}\right\}^{2}$

By determining $\mathrm{n}$ and $\mathrm{K}$ of equation (24), the non-linear stiffness matrix of contact surface is extracted.

Numerical Example

This model is modelled ANSYS software. The free-cantilever beam is based on figure (5) that beam is designed using Beam182 element. Each node of this element includes freedom degree 3. The dimensions of this beam are 30,5 , and $35 \mathrm{~mm}$ for length, thickness, and width, respectively. The clamped part is the objective of this study which tends to modeling. In joint contact surface of an excited structure, the slip and plastic penetration happens which influence on structure behavior. The slip in tangential direction and another is in vertical (normal) direction of contact surface. These replacements lead to friction and slip in tangential direction and plastic penetration is in normal direction in joint interface point. These replacements and slips can be low and high according to imposed excitation to structure. The most smoothness surface although has roughness which make friction. If excitation force amplitude increases, elastic and plastic deformations happen in these roughness that leads to slip joints in some points of interface and adhesiveness joint in some other interface points. The elastic-plastic beam element in tangential direction is used to model these properties and conditions, and non-linear elements were used in normal direction to model plastic penetration. This type of modelling makes it possible stick-slip, slip areas, and slop values to be in normal and tangential direction, and and force to be in normal and tangential directions. The objective of this modelling is to provide accurate and precise joint point of a real model according to behavior. The beam elements were modelled in tangential direction and modelled as elastoplastic and shows microscopic and microscopic roughness in a real model, and the elements in normal direction modelled by non-linear damping spring shows the plastic penetration of this roughness. The beam element behavior has been modelled using two-layer stress-strain diagram according to figure (10). 


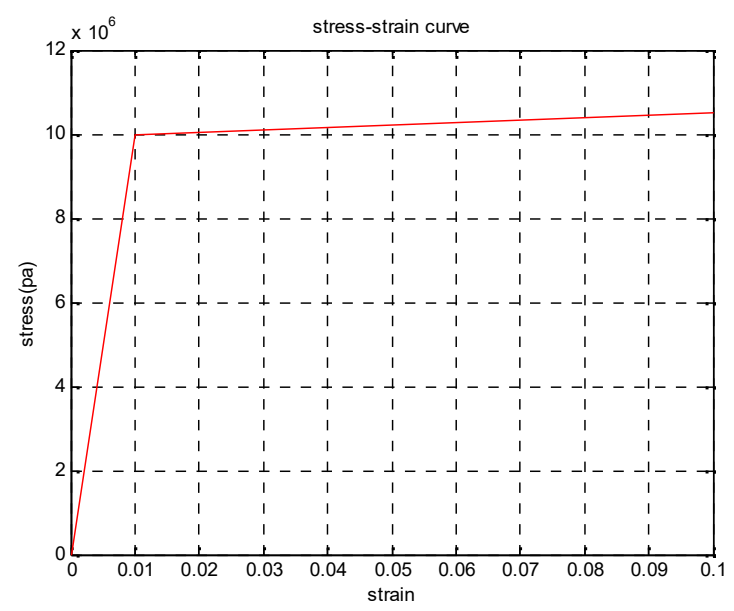

Fig.10. Two-layer stress-strain diagram

The non-linear element in normal direction of contact surface follows from thee following relation:

$$
F_{N L}=k_{s}+c_{1} v+c_{2} v^{2}
$$

In which, $k_{s}$ is spring linear stiffness, $c_{1}$ is linear damping coefficient, $c_{2}$ is non-linear damping coefficient, and $v$ is velocity in normal direction of that element.

Simulation the Linear Behavior of Joints Contact Surface by Beam-Like element In this linear modelling step, the structure is exposed to harmonic excitation with low amplitude. The non-linear properties in surface are neglected in Ansys software while harmonic analysis, and the contact surface are totally linear. Table (1) shows the flexural natural frequencies and figure (11) shows linear frequency response in logarithmic scale.

Table 1: five natural frequencies of structure first flexure

\begin{tabular}{|l|l|l|l|l|}
\hline$\omega_{1}(H z)$ & $\omega_{2}(H z)$ & $\omega_{3}(H z)$ & $\omega_{4}(H z)$ & $\omega_{5}(H z)$ \\
\hline 35.519 & 270.49 & 793.59 & 1512.8 & 2084.6 \\
\hline
\end{tabular}




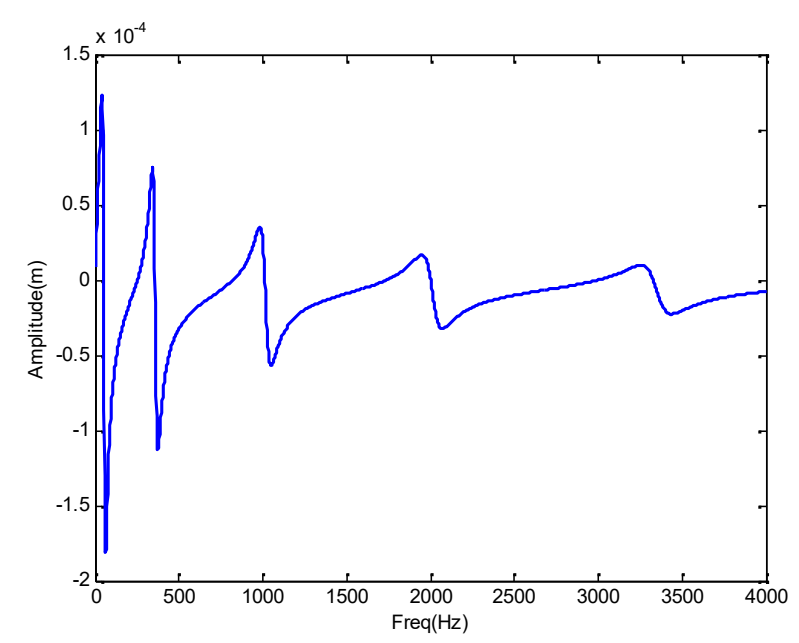

Fig.11. The linear frequency response diagram

Simulation the Non-linear Behavior of Joint Contact Surface

As it was mentioned previously, the structure non-linearity depends on the amplitude of imposed force and pre-load. In this step, the structure is exposed to excitation with more amplitude force for contact surface to show non-linear effects. The intensity of being non-linear is determined using frequency response diagram and hysteresis loop of joint elements. the non-linearity type can be estimated and the modelling accuracy is examined using these two diagrams and determines how much they get close to the real structure, then its damping and non-linear stiffness are identified and calculated. System dynamic response for $300 \mathrm{~N}$ excitation amplitude and excitation frequency of $33 \mathrm{~Hz}$, linear and non-linear frequency response with different forces amplitudes were drawn in figure (12) around the first natural frequency from left to right. 

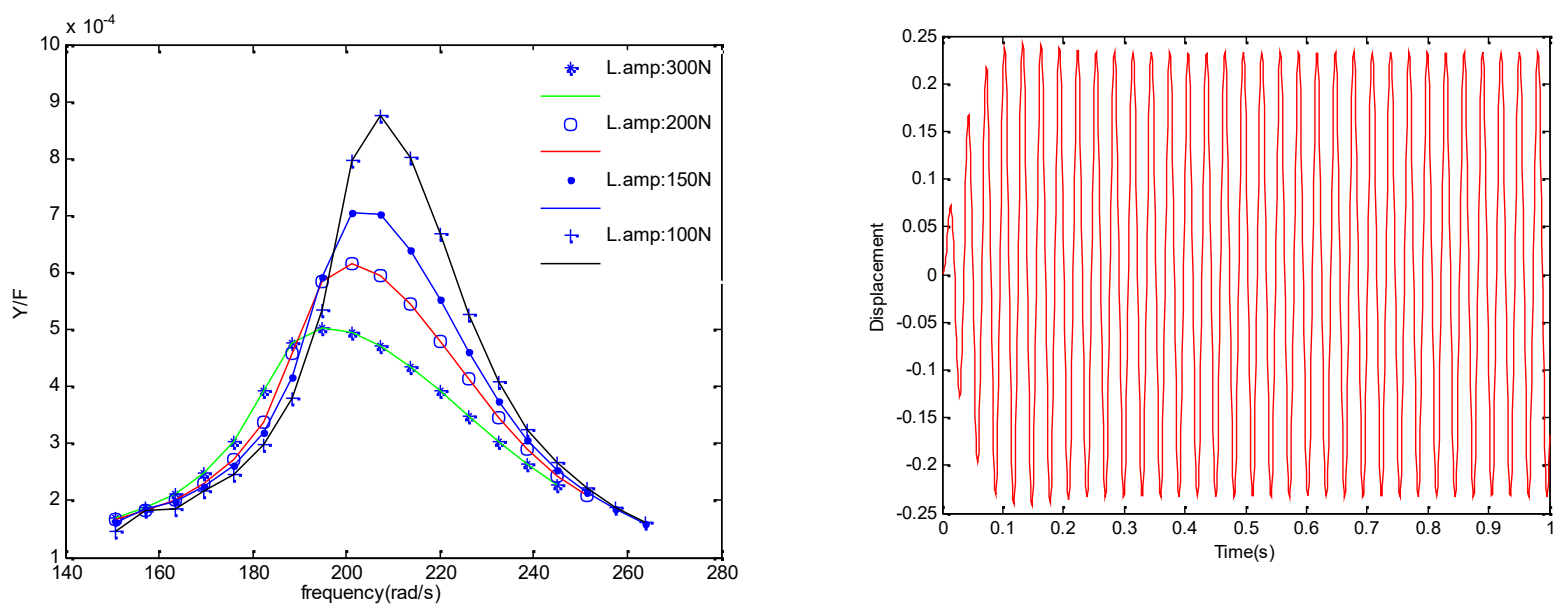

Fig.12. Left: System dynamic response for $300 \mathrm{~N}$ excitation amplitude and excitation frequency of $33 \mathrm{~Hz}$, Right: linear and non-linear frequency response with different forces amplitudes around the first natural frequency $(\mathrm{rad} / \mathrm{s})$

According to the mentioned diagram and deviation toward left, the softening phenomenon is seen that can be resulted by slip-stop element of beam in tangential direction. The reduction of amplitude of movement to force ratio shows system damping that this damping is resulted by beam elements damping and linear and non-linear damper-spring elements. Figure (13) shows hysteresis loops $\mathrm{n}$ tangential and normal direction in $300 \mathrm{~N}$ excitation amplitude and excitation frequency of $31 \mathrm{~Hz}$ from left to right. The internal area of loops shows system energy dissipation. Hysteresis loop shows tangential direction of slip and adhesion areas that curves show slip and straight lines shows adhesion.
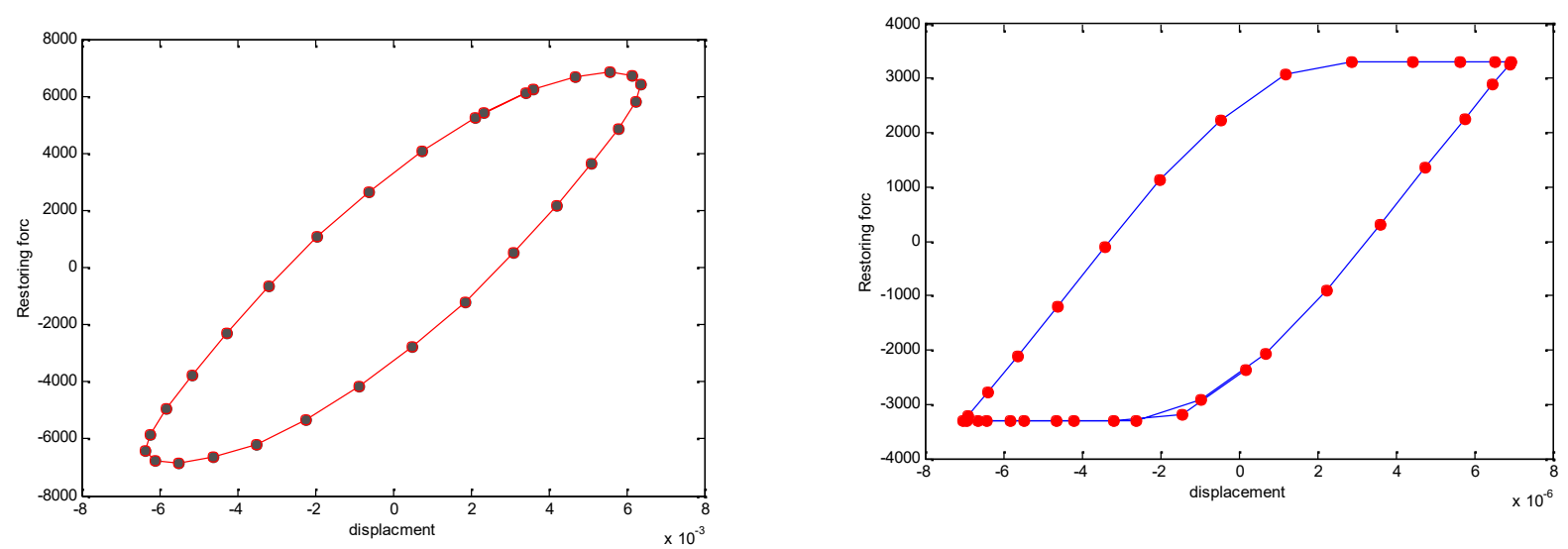

Fig.13. Right: hysteresis loop of elements in tangential direction, left: hysteresis loop of 
elements in normal direction

Another specification of accurate modeling of joint is studying energy dissipation diagram than excitation force amplitude. It is known that energy is dissipated for damping in joint interface. If the diagram of energy dissipation than excitation force amplitude is drawn in logarithmic scale, it will observe that this diagram follows a potential law relationship as $U=\alpha F^{n}$ in which $\alpha$ is constant number, $\mathrm{U}$ is energy dissipation, and $\mathrm{F}$ is excitation force amplitude, results of test show that $2<n<3$. This potential law shows damping non-linear amount in joint interface. If viscose joint damping is without non-linear damping in interface by very fixed joint, $\mathrm{n}$ is 2 , and for Colomb damping model or state that joint let friction movement in interface, the $\mathrm{n}$ will be 3 . However, for standard and tested joint in non-linear form, $2<n<3$. [19]

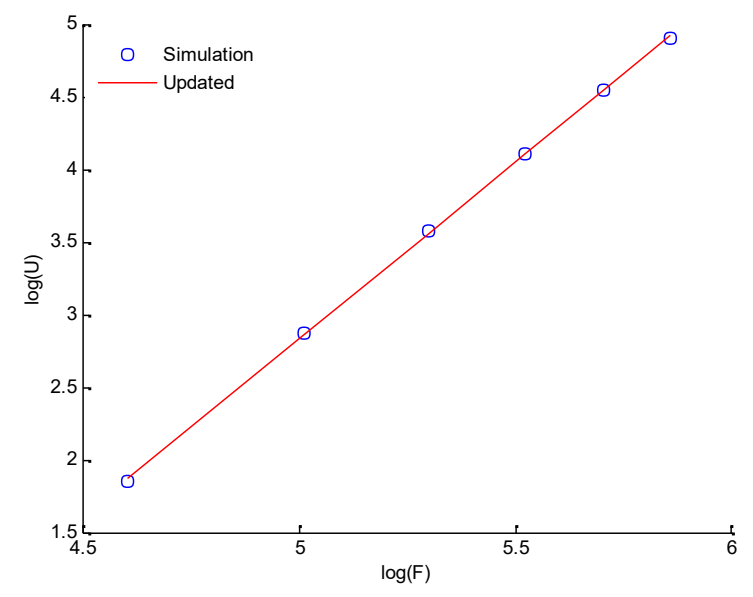

Fig.14. Energy dissipation than simulation force amplitude $(\mathrm{O})$, dissipation energy than the predicted force amplitude (-)

Diagram (14) shows energy dissipation than simulation force amplitude in comparison to logarithmic model for beam-like element model. The $\mathrm{n}$ value in determined by standardization of line by optimization method on it. The mentioned diagram is drawn for force amplitudes $F=\left[\begin{array}{llllll}100 & 150 & 200 & 250 & 300 & 350\end{array}\right]$ and excitation frequency of $30 \mathrm{~Hz}$. Table (2) shows the identified $\alpha, n$ values.

Table (2): ${ }^{\alpha, n}$ values

\begin{tabular}{ll}
\hline$n$ & $\alpha$ \\
\hline 2.4326 & $1 \mathrm{e}-4$ \\
\hline
\end{tabular}


According to the mentioned value, it can be stated about $\mathrm{n}$ that the made model is a good and referable model.

Discussion and Conclusion

This research introduces a model to simulate the contact surface of linear and non-linear joints in finite elements software. The non-linearity of a model depends on effects such as micro-slip, macro-slip, etc. the non-linear properties of beam elements in tangential direction and non-linear elements in normal directions were used to simulate behaviors of these properties. The effects of micro-slip can be examined on structure behaviors though various criterions such as frequency response diagram and hysteresis loop. The numerical simulation of this model and its solutions were done for linear and non-linear states. The softening phenomenon happens in contact surface in non-linear solution of system for presence of micro-slop which is seen in non-linear frequency response of system. In addition, the hysteresis loop of contact surface shows energy dissipation and adhesion in system. This model introduces slope between 2 and 3 showing the measurement precision.

\section{REFERENCES}

[1]. Bograd, S., Reuss, P., Schmidt, A., Gaul, L., \& Mayer, M. (2011). Modeling the dynamics of mechanical joints. Mechanical Systems and Signal Processing, 25(8), 2801-2826.

[2]. Nguyen, B. D. (2005). Modelling of frictional contact conditions in structures.Georgia Institute of Technology. MSc. thesis.

[3]. Ferri, A. A. (1995). Friction damping and isolation systems. Journal of mechanical Design, 117(B), 196-206.

[4]. Berger, E. J. (2002). Friction modeling for dynamic system simulation.Applied Mechanics Reviews, 55(6), 535-577.

[5]. Ibrahim, R. A., \& Pettit, C. L. (2005). Uncertainties and dynamic problems of bolted joints and other fasteners. Journal of sound and Vibration, 279(3), 857-936.

[6]. Iwan, W. D. (1966). A distributed-element model for hysteresis and its steady-state dynamic response. Journal of Applied Mechanics, 33(4), 893-900.

[7]. Valanis, K. C. (1971). A Theory of Viscoplasticity Without a Yield Surface, Part I: General 
Theory, Part II: Application to Mechanical Behavior of Metals. Archiwum Mechaniki Stosowanej, 23, 517-551.

[8]. Gaul, L., \& Lenz, J. (1997). Nonlinear dynamics of structures assembled by bolted joints. Acta Mechanica, 125(1-4), 169-181.

[9]. Valanis, K. C. (1971). A Theory of Viscoplasticity Without a Yield Surface, Part I: General Theory, Part II: Application to Mechanical Behavior of Metals. Archiwum Mechaniki Stosowanej, 23, 517-551.

[10]. Valanis, K. C. (1978). Fundamental consequences of a new intrinsic time measure. Plasticity as a limit of the endochronic theory (No. G-224/DME-78-01). IOWA UNIV IOWA CITY.

[11]. De Wit, C. C., Olsson, H., Astrom, K. J., \& Lischinsky, P. (1995). A new model for control of systems with friction. Automatic Control, IEEE Transactions on, 40(3), 419-425.

[12]. Dahl, P. R. (1976). Solid friction damping of mechanical vibrations. AIAA Journal, 14(12), 1675-1682.

[13]. ]. Ismail, M., Ikhouane, F., \& Rodellar, J. (2009). The hysteresis Bouc-Wen model, a survey. Archives of Computational Methods in Engineering, 16(2), 161-188.

[14]. Swevers, J., Al-Bender, F., Ganseman, C. G., \& Projogo, T. (2000). An integrated friction model structure with improved presliding behavior for accurate friction compensation. Automatic Control, IEEE Transactions on,45(4), 675-686.

[15 Lampaert, V., Swevers, J., \& Al-Bender, F. (2002). Modification of the Leuven integrated friction model structure. Automatic Control, IEEE Transactions on, 47(4), 683-687.

[16]. Mayer, M., \& Gaul, L. (2005). Modeling of contact interfaces using segment-to-segment elements for FE vibration analysis. In Proc. IMAC XXIII Conf. and Exposition on Struct. Dynamics, Soc. Expt. Mech, Paper (No. 96).

[17]. Jalali, H., Hedayati, A., \& Ahmadian, H. (2011). Modelling mechanical interfaces experiencing micro-slip/slap. Inverse Problems in Science and Engineering, 19(6), 751-764.

[18]. Logan, D. L. (2000). A first course in the finite element method using Algor. Brooks/Cole Publishing Co..

[19]. H,Jalali., H,Ahmadian.(2008). "Bolted Lap-Joints Modeling and Identification" 16th. 
Annual (International) Conference on Mechanical Engineering-ISME2008 May 14-16, , Shahid Bahonar University of Kerman, Iran.

\section{How to cite this article:}

Chonnani Mohisanzadeh A, Jalali H, Rajaei M. Linear and non-linear simulation of joints contact surface using beam-like component. J. Fundam. Appl. Sci., 2016, 8(3S), 1085-1102. 